\title{
Cost-effective and adaptable cataract surgery simulation with basic technology
}

\author{
Simerdip Kaur (iD) ${ }^{1}$, Amy-Lee Shirodkar ${ }^{2}$, Mayank A. Nanavaty $\mathbb{D}^{3,4}$ and Michael Austin ${ }^{5}$ \\ (c) The Author(s), under exclusive licence to The Royal College of Ophthalmologists 2021
}

\begin{abstract}
PURPOSE: To assess the subjective validity of a cost-effective and adaptable cataract surgery simulation technique using basic technology.

METHODS: We devised and filmed a range of simulation techniques that mimic steps of phacoemulsification cataract surgery using various "everyday" basic materials. This video was combined in a "parallel" fashion with live cataract surgery so that all steps of surgery were simulated. Subsequently, we distributed an online subjective validation questionnaire on Google Forms with the embedded simulation video in a generic invitation that was forwarded via email and/or text messages/WhatsApp messenger amongst Ophthalmologists of all grades within our regions (Kent, Surrey and Sussex, London and Wales Postgraduate Deaneries). RESULTS: Face validity: 66 (99\%) participants agreed that the explanations in the video were clear and 53 (79\%) concurred with the realistic feel of simulated technique. Instrumentation and adaptations demonstrated were deemed user friendly and conducive to replicate by $99 \%$ participants. Content validity: 60 (90\%) of participants agreed the techniques described in the video reflected the technical skills required to train cataract surgeons. Forty-nine (74\%) agreed that the simulation techniques were relevant for acquiring other generic and transferable microsurgical and manual dexterity skills.

CONCLUSIONS: We demonstrated subjective validity of our cost-effective cataract simulation technique. Our model can be used as an adjunct to intraocular and virtual reality training for cataract surgery by removing the barrier of cost and improved exposure to real instruments used in cataract surgery.
\end{abstract}

Eye (2022) 36:1384-1389; https://doi.org/10.1038/s41433-021-01644-5

\section{INTRODUCTION}

Cataract surgery is the most common surgical day case procedure carried out in the United Kingdom (UK), with over 400,000 operations conducted per year in recent years [1]. UK Ophthalmology trainees are required to complete 50 cataract operations by the end of their second year of training and 350 by the end of 7 years, with surgical simulation playing a vital role in achieving this. During their 1st year of training, Ophthalmology trainees are allocated time to complete two cataract modules on an Eyesi ${ }^{\circledR}$ Surgical (VRmagic, Mannheim, Germany) simulator, the most widely used virtual reality simulator of ophthalmic surgery in the UK. These two modules enable a novice surgeon to develop their core skills, in real time, for each step involved in routine cataract surgery including: capsulorhexis, sculpting, cracking the nucleus, removing nuclear fragments and cortical aspiration techniques. Subsequent attendance at an "Introduction to phacoemulsification microsurgical course" provided by the Royal College of Ophthalmologists is a further prerequisite before undertaking any live intraocular surgery [2]. Since becoming commonplace, this mandatory implementation of simulation training and access to an Eyesi ${ }^{\circledast}$ Surgical has been associated with a reduction in the unadjusted posterior capsule rupture rate for novice surgeons by $38 \%$ from 2009 to 2015 [3].
Further acquisition and refinement of ophthalmic microsurgical skills requires regular and repetitive practice, ideally with some trainer mentorship. The likelihood of virtual reality simulation being located conveniently to all trainees over a large geographical area around the UK is compromised by the high equipment cost involved in purchasing an Eyesi ${ }^{\circledR}$ Surgical. Evidence from other surgical specialties has identified other barriers to surgical simulation including practicality of undertaking the simulation training, trainee motivation, constraints on protected learning time and the lack of a simulation training culture [4].

To remove these barriers, one method would be to use highfidelity, low-technology and low-cost microsurgical simulation techniques for cataract surgery that are easily reproducible and accessible to all trainees and trainers. To date, whilst several such techniques have been described, very few have been validated. Recognising this deficiency and the potential benefits of such a training tool, we designed a cost-effective and easily adaptable cataract surgery simulation technique video using basic technology. We subsequently carried out a subjective validation study to primarily assess its face and content validity as well as perceptions of microsurgery simulation training in Ophthalmology.

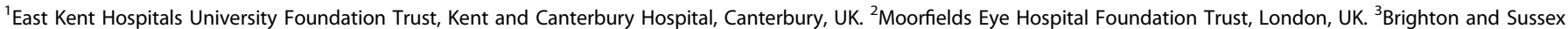
University Hospitals NHS Trust, Sussex Eye Hospital, Brighton, UK. ${ }^{4}$ Brighton and Sussex Medical School, University of Sussex, Brighton, UK. ${ }^{5}$ Singleton Hospital, Sketty, UK.

凶email: mayank.nanavaty@nhs.net
}

Received: 4 December 2020 Revised: 16 May 2021 Accepted: 14 June 2021

Published online: 25 June 2021 


\section{METHODS}

The Health Research Authority tool (http://www.hra-decisiontools.org.uk/ research/) was used to determine whether this project need an ethical approval. Based on this tool no ethics committee approval was necessary. This study was carried out in two parts.

Firstly, we considered the stages of cataract surgery and devised a range of simple drills that mimic them using various "everyday" basic materials. These were trialled by SK and MA until the most suitable materials and techniques were discovered. Live patient cataract surgery and the simulation techniques were performed by a designated surgeon (MA) on the same day and filmed by SK. The videos were then edited and combined to demonstrate the stages of routine cataract surgery together with the equivalent proposed "parallel reality" simulation drill by SK and AS so that all steps were simulated from start to finish (Video 1) (Fig. 1a-h).

In Fig. 1a, marks are drawn on the surface of the grape to indicate the main corneal incision site and two side ports. A keratome is used to make the main incision from the outside of the grape tunnelling in and a slit blade used for both side port incisions to enter the grape at a predetermined point. This exercise aims to mimic accurate placement of instruments and trajectory into the globe. In Fig. $1 \mathrm{~b}$, the top and a side section of a lozenge packet is removed. A keratome is used to pierce the aluminium foil which mimics the natural lens capsule and extend it into a flap. Then a Moorfields forceps is used to grasp and turn the flap in a continuous curvilinear fashion. In Fig. 1c, a circular slit is made on the surface of the grape. A hydrodisection cannula is placed under the skin of the grape and balanced salt solution (BSS) is injected at various angles. Subsequently in Fig. 1d, a disc of soft cheese is prepared and placed into the lozenge packet to represent the lens nucleus. The tip of a $10 \mathrm{ml}$ syringe is cut to produce a 45-degree angle. A second instrument is used to rotate and stabilise the disc whilst the syringe is advanced and withdrawn on the surface of the disc to mimic phacoemulsification. Next, in Fig. 1e, the shape of a cross is carved using a slit blade in the cheese disc to prepare it for cracking. The second instrument and syringe are aligned within the grooves made and gentle force is applied horizontally against the sides of the disc in opposite directions to crack and separate the fragments. Thereafter in Fig. 1f, the lozenge packet is filled with jelly to replicate soft lens cortex matter. Bimanual irrigation/aspiration probes are inserted through side port incisions of the lozenge packet and the jelly is aspirated. Following on in Fig. 1g, a grape is cut in half and a hollow carved in its centre. This is filled with viscoelastic material. An intraocular lens (IOL) is fashioned from a wick dyed in iodine then loaded into a viscoelastic filled cartridge using non-toothed forceps. The bevel of the injecting cartridge tip is inserted facing downwards at the main incision entry site on the grape then gently slid forward into the hollow of the grape before the plunger is pushed to release the IOL. Finally, in Fig. $1 \mathrm{~h}$, a BSS primed cannula is placed at the incision sites made earlier on the grape and the fluid injected gently to mimic corneal wound hydration.

Secondly, we devised an online subjective validation questionnaire on Google Forms with the embedded simulation video for participants to watch fully before completing the survey (see Supplementary material Table 1 for the full questionnaire). This questionnaire was distributed in a generic invitation that was forwarded via email and/or text messages/ WhatsApp messenger by the authors within their regions (Kent, Surrey and Sussex, London and Wales Postgraduate residency programmes). Ophthalmologists of all grades were included from trainees in their first and last years of training (specialty trainee year 1 (ST1) to year 7 (ST7)), Specialty and Associate Specialist (SAS) doctors (experienced senior non-consultant career grade Ophthalmologists) and consultants. We aimed to recruit equal numbers of both expert cataract surgeons (defined as those identifying as an Ophthalmology consultant or SAS doctors) as well as varying levels of novice, trainee ophthalmologists (ST1-7) to ensure a balance of views from all levels of surgical experience.

The questionnaire was distributed between the 13 and the 27 July 2020 , with a reminder sent in between. The survey was anonymous however the participants were asked to list their names at the end if they wished to be acknowledged for their contribution. The results were analysed by SK and eight duplicate responses were removed. To exclude internal bias, the authors themselves did not complete the subjective validation questionnaire.

All data were collected in Microsoft Excel Spreadsheet (Microsoft Corporation, Medmont, USA) for further analysis. Data collected included participant's demographics, level, and years of Ophthalmology experience as well as degree of agreement relating to the simulation video and microsurgery simulation training use in Ophthalmology training.
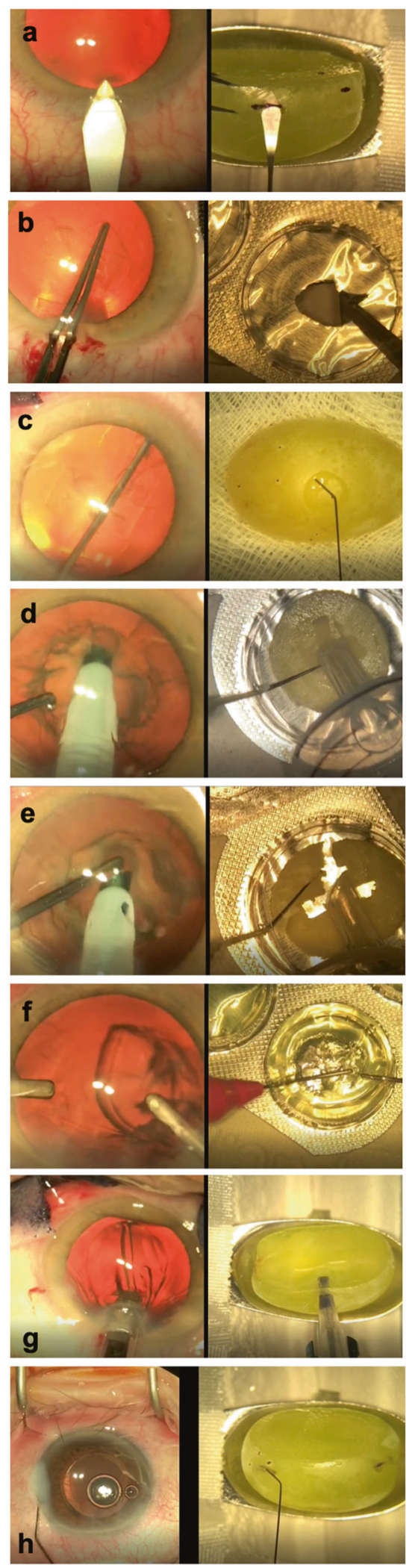

Fig. 1 Brief overview of the surgical steps. a Incision practice on grape. b Capsulorhexis on aluminium foil of lozenge packet. c Hydrodissection practice on grape. $\mathbf{d}$ Nucleus sculpting practice on cheese disc within lozenge packet. e Nucleus cracking practice on cheese disc within lozenge packet. $\mathbf{f}$ Bimanual irrigation/aspiration practice on jelly-filled lozenge packet. $\mathbf{g}$ Viscoelastic and IOL insertion practice on grape using wick dyed in iodine. $\mathbf{h}$ Corneal wound hydration practice on grape. 


\section{RESULTS}

A total of 67 people completed the survey out of 160 people whom it was sent giving a response rate of $42 \%$.

\section{Demographics and ophthalmology experience}

Table 1 summarises the demographics of the participants. We had responses from near equal numbers of expert and novice surgeons (35 experts and 32 trainees). The distribution in level of experience of participants include: 18 (27\%) ST1-3, 14 (21\%) ST4-7, 13 (19\%) SAS and 22 (33\%) consultants. Table 2 contains details of participants' previous ophthalmology experience. Duration of ophthalmology experience of participants ranged from 0-6 months to 25 years. Participants qualifying year (Ophthalmology) ranged between 1975 and 2018.

\section{Validity of the cataract simulation video}

Results of the face and content validity questionnaire are shown in Table 3. Fifty-three (79\%) participants were in agreement that the techniques demonstrated were visually realistic for simulating cataract surgery. An overwhelming majority of 66 (99\%) participants agreed that the instructions provided in the video mimicking the simulation exercises for cataract surgery were clear and that the low-tech and low-cost models used for simulation in the video would be easily obtainable and reproducible.

Sixty $(90 \%)$ participants felt the techniques described in the video were essential for developing manual dexterity and hand eye coordination skills for cataract surgery. The simulation exercises in the video were deemed an appropriate and adequate modality for teaching and learning the skills required to perform the various steps of cataract surgery by 49 (74\%) participants. A further 49 (74\%) participants believed techniques demonstrated in the video could be further refined for more experienced trainees however some believed that the techniques were more suited for novice surgeons.

Access to a surgical microscope and disposable instruments was deemed easily obtainable for 42 (63\%) participants although others were concerned that the logistical aspect of accessing theatre and the necessary equipment to undertake the simulation required concerted effort from trainees and may act as a barrier.

Table 1. Demographics of respondents.

\begin{tabular}{llll|} 
Gender & $\begin{array}{l}\text { Male } \\
{[n(\%)]}\end{array}$ & $\begin{array}{l}\text { Female } \\
{[n(\%)]}\end{array}$ & \\
& $44(66)$ & $23(34)$ & \\
\hline Handedness & Right & Left & Ambidextrous \\
\hline UK medical graduate & $63(94)$ & $1(1)$ & $3(5)$ \\
\hline & Yes & No & \\
\hline & $33(49)$ & $34(51)$ &
\end{tabular}

Fifty-seven (86\%) participants agreed that simulation training using the low-tech and low-cost techniques described in the video could increase a trainee's confidence in performing cataract surgery.

\section{Microsurgery simulation training in ophthalmology}

Table 4 summarises the participants' opinion of microsurgery simulation use in Ophthalmology training. Sixty-one (91\%) participants agreed that The Royal College of Ophthalmologists stipulate simulation training is a mandatory requirement part of the OST curriculum. Fifty-nine (89\%) participants agreed that the frequency of simulation training should occur at least 30 min once a week, using real instruments and an operating microscope.

Fifty-five (83\%) participants felt the approach taken in the video could be extended to simulate other aspects of cataract surgery, e.g. suturing, using a vitreous cutter and similarly another 50 (74\%) participants felt the approach taken in the video could be extended to assess aptitude for microsurgery, e.g. accurate placement of fine instruments, demonstration of appreciation of depth, hand-eye-microscope coordination.

The majority of consultants agreed that this low-tech simulation could act as a supplementary aid to performing surgery on real patients, and it would allow novice trainees to familiarise themselves with instruments actually used during cataract surgery. However, some commented that a closed system of simulation training would be more beneficial than the open and deconstructed system we have demonstrated.

\section{DISCUSSION}

Simulation in surgical skills must be high-fidelity, cost-effective, and feasible in order to be worthwhile and we believe all three criteria are fulfilled by our model [5]. Additionally, we aimed to address the two main elements of subjective validity-face and content validity, through the questionnaire $[6,7]$.

Face validity in the context of this work is a measure of how well the simulation techniques depicted in the video represents the visual experience of "live patient" cataract surgery $[6,7]$. This was achieved as $99 \%$ of participants felt the explanations in the video were clear and over three quarters (79\%) concurred with the realism of the simulated technique to mimic live cataract surgery. Instrumentation used and adaptations demonstrated were deemed user friendly and conducive to replicate by $99 \%$ participants.

Content validity refers to the extent by which the simulation techniques accurately correspond to the level of knowledge and competence associated with performing the tasks in the simulation training $[6,7]$. This includes the "feel" (of real instruments moving in ways akin to "live patient" surgery) to accompany the "look" of the simulation and more visual aspects of hand-eye coordination. This was achieved as $90 \%$ of participants agreed that

Table 2. Previous Ophthalmology experience.

Did you have experience of suturing or assisting with or performing surgery in any of your posts as a doctor since qualifying and prior to starting ophthalmology?

How many years have you been a doctor in ophthalmology?

How many entire cataract operations have you done personally, i.e. without any other [supervising] surgeon having to do any part of the procedure?

If you have answered the previous question as fewer than 101 cases, how may cataract operations have you undertaken, including those where a supervisor did part of the procedure?

\begin{tabular}{llll}
$\begin{array}{l}\text { Yes } \\
{[n(\%)]}\end{array}$ & $\begin{array}{l}\text { No } \\
{[n(\%)]}\end{array}$ & & \\
\hline $52(78)$ & $15(22)$ & & \\
$<3$ years & $3-5$ years & $5-10$ years & $>10$ years \\
\hline $18(27)$ & $7(10)$ & $17(25)$ & $25(38)$ \\
$0-100$ & $101-200$ & $>200$ & $>2000$ \\
$15(23)$ & $7(10)$ & $24(31)$ & $21(36)$ \\
$0-10$ & $11-50$ & $51-100$ & $101-150$ \\
\hline $4(26.7)$ & $3(20)$ & $7(46.7)$ & $1(6.7)$
\end{tabular}




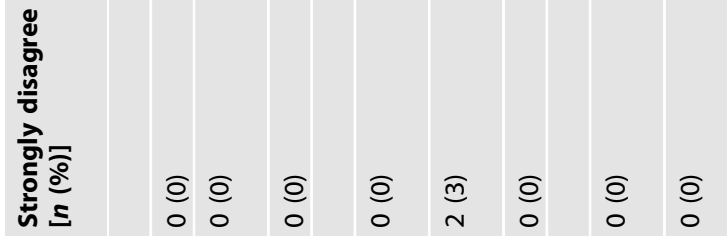

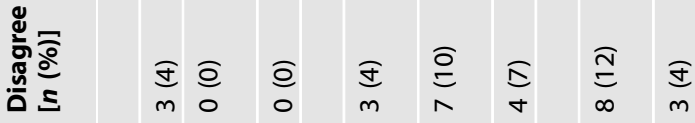

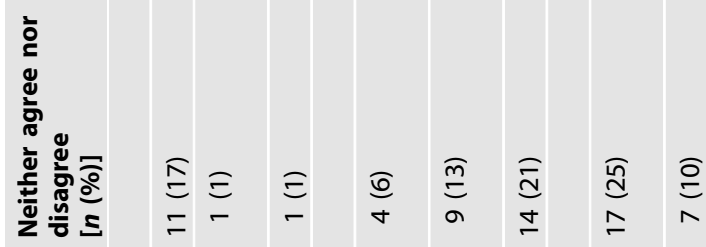

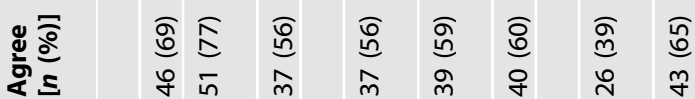

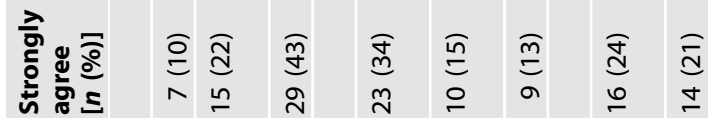

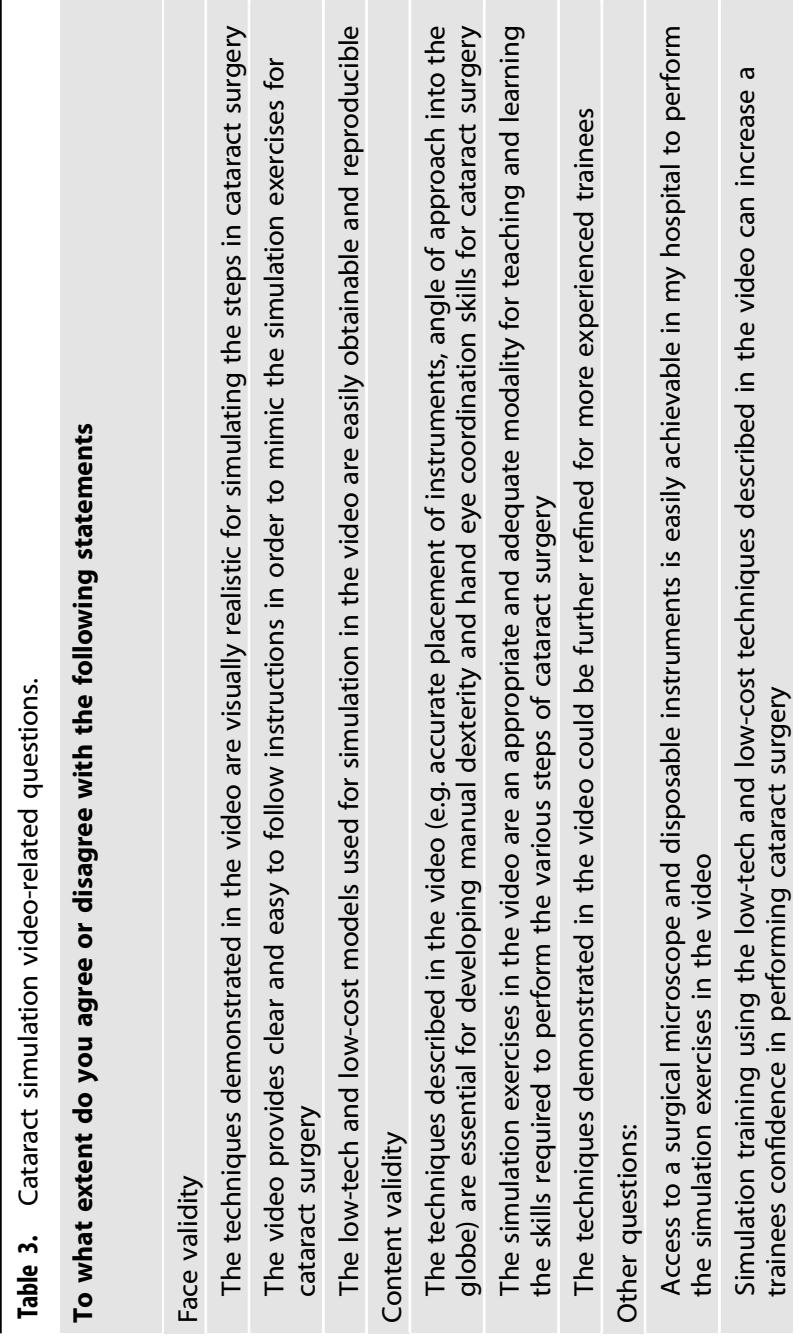

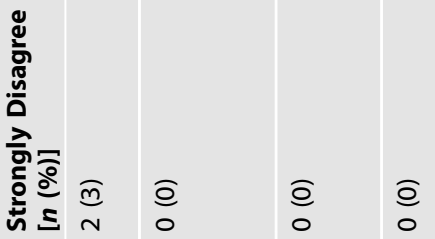

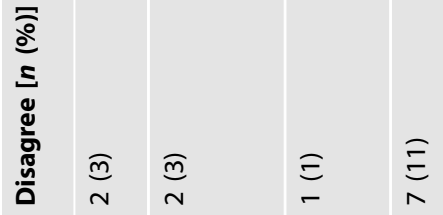

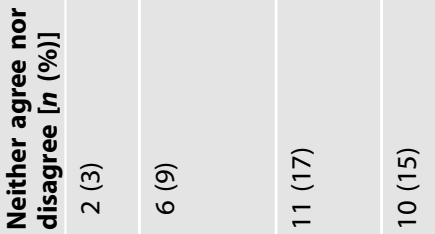

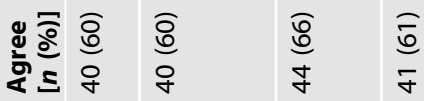
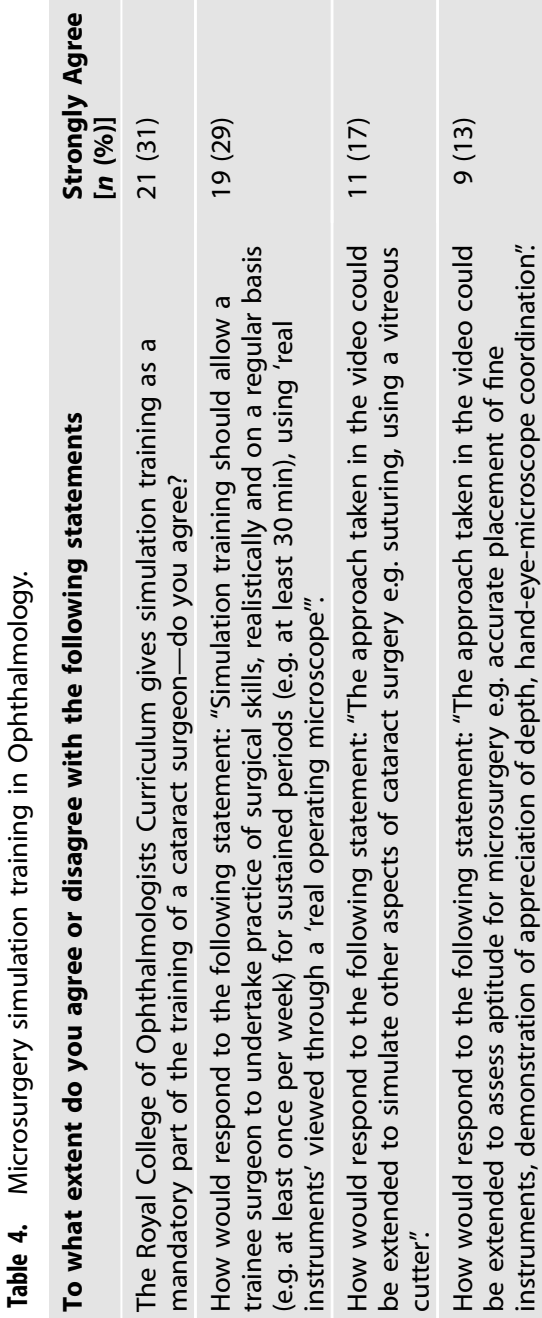
1388

the techniques described in the video reflected the technical skills required to train cataract surgeons. In total, $74 \%$ agreed that the simulation techniques were relevant for acquiring other generic and transferable microsurgical and manual dexterity skills, whilst $73 \%$ believing that more experienced trainees could benefit from refinement of the techniques. This indicates the substance behind our simulation techniques are of high quality and provide a good foundation to build on. Therefore, this study positively validated the use of a low-tech, low-cost cataract simulation video produced by the study authors.

In terms of adaptability, the results from our study prove that the simulation techniques are deemed high-fidelity as the technique shown is repeatable, bears resemblance to and corresponds well with the exactness to live cataract surgery. One Consultant commented, "I have encountered various DIY suggestions over the years, but usually in disparate pieces. I haven't seen such a well-produced video clearly linking the steps of cataract surgery to an achievable simulation." Notably however two participants commented, "The material used to simulate the IOL and the eye could be refined," and "The depth perception and the way the lens material behaves during phacoemulsification is not well simulated in this video." (See Supplementary material Table 2 for the full list of free text comments). We have not performed concurrent or construct validity for this simulation video and these assessments would be a practical option as the next step in validating our model.

In a recent systematic review of simulation-based training modalities in Ophthalmology, several wet lab models for cataract surgery were identified however only two had the clearest evidence for content validity [8]. One was a pig's eye model filled with chestnuts for simulating phacoemulsification and another was a rabbit's eye model for capsulorhexis training [8]. As for cataract dry lab simulations, one study using a methacrylate support and aluminium foil for capsulorhexis simulation was tested in a randomised controlled trial [9]. The authors demonstrated improved capsulorhexis performance in those who trained using the synthetic model compared to those who did not thus overall reducing the number and cost of acquiring cadaveric animal eyes for training purposes [9]. There was no other validity parameter under study besides the transfer effects described.

Our low-tech model of simulation would involve the trainee to locally purchase 5 grapes, 1 small cheese disc, a packet of 12 lozenges and instant jelly mix, which in our example totalled approximately $£ 1.50$ or $\$ 1.96$. They would also need an operating microscope, phacoemulsification machine for irrigation and aspiration and disposable surgical instruments-see Supplementary material Table 3 for full list of instruments. This is in stark contrast to the Eyesi ${ }^{\circledR}$ Surgical simulator which has an estimated purchase cost of $£ 100,000$ or $\$ 130,455$ [10]. This limits the availability of the device thus necessitating travelling long distances to access it. Other cost-effective options for cataract surgery simulation include the use of synthetic simulation eyes from Phillips Studio ${ }^{\odot}$ or Simulated Ocular Surgery ${ }^{\circ}$ that cost $\sim £ 20-$ $£ 40$ or $\$ 26-\$ 52$ per eye and cadaveric animal eyes from Wetlab $\mathrm{Ltd}^{\odot}$ which are slightly cheaper at between $£ 5-£ 10$ or $\$ 6.50-\$ 13$ per eye [10-13]. The use of animal tissue poses its own challenges in procurement, storage and correct disposal afterwards thus may be least preferred especially due to time constraints.

There is a critical need for low-tech and low-cost simulation models now more than ever given the ongoing coronavirus (COVID-19) pandemic, which has affected access to simulation centres and routine, supervised cataract surgery [14]. Within the field of Ophthalmology, an online survey distributed to trainees in 32 countries revealed that $74.6 \%$ experienced a reduction of $>75 \%$ of surgical training and practice and $56 \%$ reported the absence of simulation-based tools for training in their hospital during the pandemic [15]. Whilst some countries have recovered from the initial peak of the pandemic and are now attempting to resume pre-COVID-19 levels of clinical activity, their healthcare systems are now faced with a backlog resulting in a high pressure environment to deliver these services which will inevitably compromise training needs further going forward. Furthermore, the existing challenges of outsourcing of routine cataract cases away from training hospitals has an additional negative impact on trainees' access to surgical training $[16,17]$. These gaps in surgical experience could be filled using cataract simulation should be strongly considered for both the novice surgeon as well as the expert having had time away from their surgical responsibilities.

There are limitations of this study. Firstly, we could only achieve a response rate of $42 \%$ but this response rate is akin to the response rates received in other similar studies (e.g. 21\% [14], 24.1\% [18], 27\% [19], 41\% [20]). This is typical of questionnaire-based studies and is known to increase the risk of response and selection bias. However, we also postulate that most units had resumed some level of elective cataract surgery by the time this study questionnaire was carried out (13-27 July 2020) which could explain the low response rate. Thirdly, we recognise the presence of subjective bias amongst the participants who voluntarily contributed to this study and that these individuals were more likely to have their surgical training negatively impacted by the pandemic thus were more receptive towards our proposed simulation training video. The authors agreed to modify the original simulation techniques model if more than $15 \%$ of respondents disagreed with any aspect of it however this was not required.

In conclusion, we have successfully demonstrated subjective validity of our cost-effective cataract simulation technique acceptable to UK based Ophthalmology trainees (years 1-7), SAS and consultants. Simulation has previously been shown to improve complication rates in novice surgeons and should be strongly considered to fill gaps in surgical experience, such as that faced by most cataract surgeons around the world during the COVID-19 pandemic. We hope that we have been able to provide creative options for trainees to practice their surgical skills frequently and regularly. This technique could be an adjunct to intraocular and virtual reality training for cataract surgery removing the barrier of cost and improved exposure to real instruments used in cataract surgery.

\section{SUMMARY}

What was known before

- Multiple validation studies have been conducted on virtual reality simulators for cataract surgery, however their use is often limited by high cost and limited availability.

- Non-virtual reality cataract simulation studies are few and far in between with limited data on their validity.

What this study adds

- We have subjectively validated our simulation training model which includes all the steps of cataract surgery from start to finish in a cost-effective, adaptable, high-fidelity and acceptable manner.

- Our model of cataract surgery simulation with basic technology can be carried out frequently, regularly and locally in every unit for training purposes without significant costs.

\section{REFERENCES}

1. National Institute for Health and Care Excellence [Internet]. United Kingdom. Cataracts in adults: management NICE guideline [NG77]. 2017. https://www.nice. org.uk/guidance/ng77. Accessed 5 Aug 2020. 
2. Royal College of Ophthalmologists [Internet]. United Kingdom. Microsurgical skills course changes for 2019. 2019. https://www.rcophth.ac.uk/2019/06/ microsurgical-skills-course-changes-for-2019/. Accessed 5 Aug 2020.

3. Ferris JD, Donachie PH, Johnston RL, Barnes B, Olaitan M, Sparrow JM. Royal College of Ophthalmologists' National Ophthalmology Database study of cataract surgery: report 6 . The impact of EyeSi virtual reality training on complications rates of cataract surgery performed by first and second year trainees. $\mathrm{Br} J$ Ophthalmol. 2020;104:324-9.

4. Hosny SG, Johnston MJ, Pucher PH, Erridge S, Darzi A. Barriers to the implementation and uptake of simulation-based training programs in general surgery: a multinational qualitative study. J Surg Res. 2017;220:419-26.e2.

5. Atesok K, Hurwitz S, Anderson DD, Satava R, Thomas GW, Tufescu T, et al. Advancing simulation-based orthopaedic surgical skills training: an analysis of the challenges to implementation. Adv Orthop. 2019;2019:2586034.

6. Gallagher AG, Ritter EM, Satava RM. Fundamental principles of validation, and reliability: rigorous science for the assessment of surgical education and training. Surg Endosc. 2003;17:1525-9.

7. Alsalamah A, Campo R, Tanos V, Grimbizi G, Van Belle Y, Hood K, et al. Face and content validity of the virtual reality simulator 'ScanTrainer ${ }^{\circledR '}$. Gynecol Surg. 2017; $14: 18$.

8. Lee R, Raison N, Lau WY, Aydin A, Dasgupta P, Ahmed K, et al. A systematic review of simulation-based training tools for technical and non-technical skills in ophthalmology. Eye (London). 2020;34:1737-59.

9. Abellan E, Calles-Vazquez MC, Cadarso L, Sanchez FM, Uson J. Design and validation of a simulator for training in continuous circular capsulotomy for phacoemulsification. Arch Soc Esp Oftalmol. 2013;88:387-92.

10. The Royal College of Ophthalmologists [Internet] United Kingdom. Simulation information. 2015. https://www.rcophth.ac.uk/wp-content/uploads/2015/06/ Web-doc-simulation-information.pdf. Accessed 10 Aug 2020.

11. Phillips Studio-Ophthalmic Simulated Surgery [Internet] United Kingdom. Tactile synthetic simulation eyes-cataract eyes. http://www.phillipsstudio.co.uk/ eyes.html. Accessed 10 Oct 2020.

12. Simulated Ocular Surgery [Internet] United Kingdom. Products-cataracts. http:// simulatedocularsurgery.com/product-category/eyes/cataracts/. Accessed $10 \mathrm{Oct}$ 2020.

13. Wetlab [Internet] United Kingdom. Tissue supply. Wetlab medical tissue. https:// www.wetlab.co.uk/instruments_and_supplies/tissue_supply.aspx. Accessed 10 Oct 2020.

14. Ellison EC, Spanknebel K, Stain SC, Shabahang MM, Matthews JB, Debas HT, et al. Impact of the COVID-19 pandemic on surgical training and learner well-being: report of a survey of general surgery and other surgical specialty educators. J Am Coll Surg. 2020;231:613-26.

15. Ferrara M, Romano V, Steel DH, Gupta R, lovino C, van Dijk EHC, OphthaTraining Group, et al. Reshaping ophthalmology training after COVID-19 pandemic. Eye (London). 2020;34:2089-97.

16. Wijetilleka S, Yeo CM, Roberts A, Sengupta R. The impact of outsourcing on cataract training in the UK. Eye (London). 2017;31:827.

17. Barsam A, Heatley CJ, Sundaram V, Toma NM. A retrospective analysis to determine the effect of independent treatment centres on the case mix for microsurgical training. Eye (London). 2008;22:687-90.

18. Dean WH, Grant S, McHugh J, Bowes O, Spencer F. Ophthalmology specialist trainee survey in the United Kingdom. Eye (London). 2019;33:917-24.
19. Stairs J, Bergey BW, Maguire F, Scott S. Motivation to access laparoscopic skills training: results of a Canadian survey of obstetrics and gynecology residents. PLoS ONE. 2020;15:e0230931.

20. Le K, Bursztyn L, Rootman D, Harissi-Dagher M. National survey of Canadian ophthalmology residency education. Can J Ophthalmol. 2016;51:219-25.

\section{ACKNOWLEDGEMENTS}

We would like to thank all of our colleagues who participated in our subjective validation study. Their names are as follows in no particular order: Hasan Naveed, Andrew Swampillai, Chris Ashton, Rohini Yardy, ljaz Sheikh, Minas Georgopoulos, Sleiman Abou Latif, Tariq Saboor, Nadia Azad, Ahsan Bhatti, Rashid Zia, Mo Majid, Syed T Hasan, S A Aftab, Rosina Zakri, Sayali Pradhan, Khurram Rahman, Esther Papamichael, Vidushi Golash, Asad Zaheer, Kashif Ali, Muhammad Imran Asif, Stafford Sansome, Moloy Dey, Vishal Shah, Hajra Arshad Malik, M Ayaz Asghar, Hanbin Lee, Sam Kanavati, Colm McAlinden, Nikhil Kaushik, Thevamalar Puspanathan, Doa'a Kerwat, Sarju Athwal, Kam Balaggan, Jai Shankar, Scott Cutting, Dharshana Ramanathan, Tariq Mohammad, Alexander Chiu, Fatimah Z Javaid, Eleanor McCance, Sundas Maqsood, Laura Drayer Turner, Masara Laginaf, Pratibha Veeramani, Mikes Nomikarios, Gwyn Williams, Bhavin Patel and Nick Kopsachilis.

\section{AUTHOR CONTRIBUTIONS}

SK and ALS: conceptualisation, conducting the study, data collection and analysis, manuscript drafting/approval, MAN: design of the study, analysis of data, manuscript drafting/approval, supervision, MA: conceptualisation, conducting the study, analysis of data, manuscript drafting/approval, supervision.

\section{COMPETING INTERESTS}

SK: Joint founder of EyeducationUK Ltd. and has received educational grants from Scope Ophthalmics, Altomed Ltd, Altacor and Thea Pharmaceuticals Limited. ALS Joint founder of EyeducationUK Ltd and has received educational grants from Scope Ophthalmics, Altomed Ltd, Altacor and Thea Pharmaceuticals Limited. MAN: Research grants from Alcon Laboratories, USA; European Society of Cataract \& Refractive Surgery; Johnson \& Johnson, USA; Rayner Intraocular lenses, UK. Lecture fees from Alcon Laboratories, USA. Consultant to Hoya \& NuVision Biotherapies, UK. Travel grant from Alcon Laboratories, USA and Bausch \& Lomb, USA. MA: none.

\section{ADDITIONAL INFORMATION}

Supplementary information The online version contains supplementary material available at https://doi.org/10.1038/s41433-021-01644-5.

Correspondence and requests for materials should be addressed to M.A.N.

Reprints and permission information is available at http://www.nature.com/ reprints

Publisher's note Springer Nature remains neutral with regard to jurisdictional claims in published maps and institutional affiliations. 